\title{
Wieder ein Sieg der Angst? Das zweite irische Referendum über den Lissabon-Vertrag in der Analyse
}

\author{
Wolf J. Schünemann*
}

\begin{abstract}
Als Sieg der Angst bezeichnete Joachim Schild in einer früheren Ausgabe der ,integration" den Ausgang des französischen Referendums über den Vertrag über eine Verfassung für Europa (Verfassungsvertrag) im Jahr 2005 und führte die mehrheitliche Ablehnung des Vertragswerks in einer überzeugenden Analyse auf die zum damaligen Zeitpunkt spürbare tiefe sozioökonomische Verunsicherung innerhalb der französischen Gesellschaft zurück. ${ }^{1}$ Wenn die vorliegende Untersuchung nun ganz bewusst ein anderes augenscheinlich mit sozioökonomischen Zukunftsängsten beladenes Referendum unter ähnlicher Fragestellung in den Blick nimmt, mag dies aus europapolitischer Hinsicht zunächst fernliegen. Denn schließlich ging das zu betrachtende zweite irische Referendum über den Lissabon-Vertrag am 2. Oktober 2009 mit einer überraschend deutlichen Mehrheit von 67,1 Prozent (bei einer Wahlbeteiligung von 59 Prozent) für die Ratifizierung aus.

Natürlich bewirkte diese erfolgreiche Abstimmung eine große europapolitische Erleichterung, die umso größer war, als die Iren im Juni 2008 in einem ersten Referendum über den Lissabon-Vertrag mehrheitlich (53,4 Prozent bei einer Beteiligung von 53,1 Prozent) ihre Zustimmung zur Ratifizierung verweigert und der Europäischen Union auf ihrem Reformpfad eine neuerliche schmerzhafte Niederlage zugefügt hatten. Im Herbst 2009 spitzte sich die Lage demgegenüber noch einmal zu und alle Augen schauten nun erst recht auf Irland, weil nahezu alle übrigen Mitgliedstaaten ihre - ausnahmslos parlamentarischen - Ratifizierungsverfahren erfolgreich abgeschlossen hatten. Die irische Ratifizierung stellte somit, wenn auch nicht die letzte, so doch die problematischste verbliebene Hürde auf dem Weg zur konstitutionellen Reform dar.

Vor diesem Hintergrund kommt der Frage, welche Faktoren einen derart sprunghaften Stimmenzuwachs von gut 20 Prozent (oder knapp 500.000 Stimmen) für das ,Ja“ innerhalb von 16 Monaten bewirken konnten, eine große Bedeutung zu. Viele Anzeichen sprechen für die Hypothese, dass - nun offenbart die Anknüpfung an die Formel von Schild ihren tiefer liegenden Sinn - die dramatische Abwärtsentwicklung der irischen Wirtschaft im Kontext der Finanz- und Wirtschaftskrise mit Massenarbeitslosigkeit, katastrophaler Haushaltslage und bevorstehenden Sozialkürzungen als dominanter Erklärungsfaktor für den wirkungsvollen Stimmungswandel zugunsten des Lissabon-Vertrags angesehen werden kann.
\end{abstract}

\section{Das unvermeidbare Referendum...}

Nach dem Scheitern des Verfassungsvertrags an den Voten der Franzosen und Niederländer im Frühsommer 2005 und der selbst verordneten Phase der Reflexion im krisenhaften Reformprozess war die zuallererst im Verfassungsbegriff sich manifestierende föderalistische Ambition im Frühjahr 2007 einem pragmatischeren Ansatz gewichen. Die Europapolitiker besannen sich notgedrungen auf den vertrauten Modus konstitutioneller Reformen und

\footnotetext{
* Wolf J. Schünemann, M.A., Wissenschaftlicher Mitarbeiter, Abteilung Politikwissenschaft, Institut für Sozialwissenschaften, Universität Koblenz-Landau, Campus Landau.

1 Joachim Schild: Ein Sieg der Angst - das gescheiterte französische Verfassungsreferendum, in: integration 3/2005, S. $187-200$.
} 
wählten das entsprechende Instrument eines Reformvertrags. Ferner wurden auch die übrigen Elemente konstitutioneller Symbolik, also Flagge, Hymne, Leitspruch, Gesetzesbegriff und Außenministertitel etc., aus dem Dokument gestrichen.

Während sich alle übrigen Mitgliedstaaten nicht zuletzt wegen dieser reduzierten Ambitionen mit der Verabschiedung der jeweiligen Ratifizierungsgesetze per Parlamentsentscheid begnügen konnten, war die Durchführung einer Volksabstimmung in Irland schon 2008 unausweichlich. Denn die irische Verfassung (Bunreacht na hÉireann) schreibt in Art. 46 ein obligatorisches Referendum für Verfassungsänderungen vor. Damit war Irland ,from the outset a referendum country“2 , das heißt seit seinem 1972 per Volksabstimmung erlassenen Beitrittsgesetz. Bis heute ist jede Vertragsreform per Referendum ratifiziert worden. So war die Durchführung eines zweiten Referendums 2009 auf der einen Seite unausweichlich, sollte der Vertrag von Lissabon doch noch in Kraft treten können - und darauf drangen die Partnerstaaten auf EU-Ebene -, auf der anderen Seite konnte Premierminister Brian Cowen dem Volk schwerlich das exakt gleiche Verhandlungspaket zur Abstimmung vorlegen.

\section{...zum (un-)veränderbaren Vertrag}

Die irische Regierung stand nach dem gescheiterten ersten Referendum also tatsächlich vor einem Dilemma. Der Druck wurde dadurch verstärkt, dass die Partner auf europäischer Ebene aktive Schadensbegrenzung von ihr erwarteten. Dabei konnte der Gegenstand für ein zweites Referendum, der Reformvertrag, ${ }^{3}$ im Grunde nicht verändert werden, ohne dass dies eine neuerliche Ratifizierung durch alle übrigen Staaten erfordert hätte. Wie sollte die Regierung nun also dem Abstimmungsergebnis Rechnung tragen und für eine zweite Volksabstimmung einen neuen , deal' aushandeln, wenn der Vertrag selbst nicht geändert werden konnte?

Eine Lösung wurde mit den sogenannten Garantien gefunden. Dies ist die informelle Bezeichnung für die von den 27 Staats- und Regierungschefs auf ihrem Gipfeltreffen am 19. Juni 2009 getroffene Vereinbarung. ${ }^{4}$ Sie umfasst drei Dokumente: zum Ersten einen Beschluss der Staats- und Regierungschefs von der Qualität eines Abkommens im internationalen Recht. Dieser enthält in seinen drei Abschnitten jeweils eine Klarstellung zu konkreten Bedenken der irischen Bevölkerung, wie sie sich als wesentliche Gründe für die Ablehnung des Vertrags im ersten Referendum herausgestellt hatten. Schon im Juli/August 2008 hatte das Meinungsforschungsinstitut „Millward Brown IMS“ im Auftrag der Regierung umfangreiche Befragungen durchgeführt. ${ }^{5}$ Als mögliche Ursachen für die Ablehnung des ersten Referendums ermittelt und entsprechend von den Garantien aufgegriffen wurden demnach sozialethische Themen (Recht auf Leben, Familie und Bildung; Abschnitt A), die Steuerpolitik (Abschnitt B) sowie schließlich die außenpolitische Neutralität Irlands (Abschnitt C). Diese drei Kernbereiche irischer Politik und nationaler Identität seien, so die Klarstellungen, von den Neuerungen des Lissabon-Vertrags unberührt und verblieben in der souveränen Entscheidungsgewalt des irischen Staates. ${ }^{6}$

2 Brigid Laffan/Jane O'Mahony: Ireland and the European Union, Basingstoke u.a. 2008, S. 24.

3 Genau genommen, war der Gegenstand der Referenden jeweils die ,28th Amendment of the Constitution Bill“", deren Wortlaut sich zwischen den Referenden zwar verändert hatte; der Kerngehalt aber blieb bestehen: die Ratifizierung des Lissabon-Vertrags.

4 Rat der Europäischen Union: Tagung des Europäischen Rates in Brüssel vom 18./19. Juni 2009. Schlussfolgerungen des Vorsitzes, Dok. 11225/2/09, S. 17-23.

5 Millward Brown IMS: Post Lisbon Treaty Referendum Research Findings, September 2008; darauf aufbauend: Richard Sinnott/Johan A. Elkink/Kevin O'Rourke/James McBride: Attitudes and Behaviour in the Referendum on the Treaty of Lisbon, UCD Geary Institute University College Dublin, 06.03.2009.

6 Rat der Europäischen Union: Tagung des Europäischen Rates in Brüssel vom 18./19. Juni 2009. Schlussfolgerungen des Vorsitzes, Dok. 11225/2/09, S. 17-19. 
Zum Zweiten und nicht in Form eines rechtsverbindlichen Beschlusses, enthielt die Vereinbarung des Europäischen Rates eine „Feierliche Erklärung zu den Rechten der Arbeitnehmer, zur Sozialpolitik und zu anderen Angelegenheiten", 7 die vornehmlich der in der ersten Referendumsdebatte ebenfalls lautstark artikulierten Kritik an der angeblich neoliberalen Ausrichtung des Vertragswerks begegnen sollte. Zum Dritten umfasste die Vereinbarung eine weitere nationale Erklärung Irlands, ${ }^{8}$ die noch einmal die Sonderrolle des irischen Staates bei der Beteiligung an Maßnahmen im Rahmen der Außen- und Sicherheitspolitik bekräftigte.

Stellten die genannten Elemente der Vereinbarung lediglich für die irischen Bürger relevante Klarstellungen dar, war schon zuvor, nämlich anlässlich des Europäischen Rates im Dezember 2008, die Rücknahme einer institutionellen Neuerung des Lissabon-Vertrags vereinbart worden, die in der ersten Referendumsdebatte ebenfalls verbreitet kritisiert worden war und in der Tat alle EU-Mitgliedstaaten betrifft: die Reduktion des Kommissionskollegiums. ${ }^{9}$ Mit diesem Zugeständnis an die irischen Bedenken war es den Ratifizierungsbefürwortern 2009 nicht nur möglich, dahingehend zu argumentieren, dass auch mit dem Vertrag von Lissabon Irland immer einen Vertreter im Kommissionskollegium stellen würde. Vielmehr konnte sich das , Ja'-Lager dank des Junktims zwischen dem in Aussicht gestellten Beschluss und dem Inkrafttreten des Lissabon-Vertrags und eingedenk der Tatsache, dass auch der Nizza-Vertrag eine Reduktion des Kommissionskollegiums schon ab 2009 verbindlich vorsah, sogar eines gleichsam umgekehrten ,Kommissionsarguments ' bedienen. Statt auch konnte es heißen: „Nur mit Lissabon behalten wir unseren Kommissar“.

Die Wirkung der Garantien einschließlich der Regelung betreffend das Kommissionskollegium war freilich nicht so groß, als dass sich Argumente zu Themen wie Abtreibung, Sterbehilfe, Steuerharmonisierung und Gefährdung der irischen Neutralität für das ,Nein'-Lager nun 2009 quasi verboten hätten. Im Gegenteil, sie wurden von den jeweiligen Sprechern erneut artikuliert und mit einem generellen Misstrauen an der ohnehin unpopulären Regierung verbunden. Ebenso wie jeder Vertragsartikel waren also auch die auf europäischer Ebene getroffenen Vereinbarungen in der nationalen Debatte den gegensätzlichen Deutungen der beiden Lager ausgesetzt und wurden in entsprechend divergierender Weise interpretiert.

\section{Zum diskursanalytischen Vorgehen}

Referendumsforschung findet in der Regel mit den Mitteln der Wahlsoziologie statt. Entsprechend werden Daten generiert, aggregiert und/oder verwendet, die Aussagen über das Wählerverhalten in einer gewissen Abstimmung erlauben. So ist es auch 2008 in den bereits zitierten Studien ${ }^{10}$ geschehen und auch für das irische Referendum 2009 liegen bereits Ergebnisse einer Eurobarometer-Befragung vor. ${ }^{11}$ Die Meinungen der Befragten, wie sie im Rahmen derartiger Untersuchungen gesammelt werden und die wir für unsere Zwecke im konkreten Fall ebenso gut als subjektives Wissen über den Vertrag von Lissabon, die Europäische Union etc. auffassen können, sind dabei alles andere als voraussetzungsfrei. Das je subjektive Wissen ist keine individuelle Angelegenheit, sondern gesellschaftlichen Kon-

7 Ebenda, S. 20-21.

8 Ebenda, S. 22-23.

9 Rat der Europäischen Union: Tagung des Europäischen Rates vom 11./12. Dezember 2008 in Brüssel. Schlussfolgerungen des Vorsitzes, Dok. 17271/1/08, S. 2.

10 Millward Brown IMS: Post Lisbon Treaty Referendum, 2008; Sinnott/Elkink/O'Rourke/McBride: Attitudes and Behaviour, 2009.

11 Vgl. Europäische Kommission: Lisbon Treaty Post-Referendum Survey Ireland 2009, Flash Eurobarometer 284, Oktober 2009. 
struktionsprozessen unterworfen. Indem die vorliegende Untersuchung das Referendum weniger als Wahlakt denn als Wahlkampf in den Blick nimmt und die entsprechende, der Abstimmung vorangegangene Debatte mit Reiner Keller als mehrmonatigen Zusammenhang einer ,inhaltlich-thematisch bestimmte[n], institutionalisierte[n] Form der Textproduktion" 12 versteht, gibt sie unweigerlich den ersten Teil ihrer theoretisch-methodologischen Abstammung zu erkennen und verortet sich im radikalkonstruktivistischen Umfeld der Wissenssoziologie. ${ }^{13}$

Die Strukturen einer Wissensordnung anhand von Diskursen freizulegen, erlaubt auf der anderen Seite die an den Schriften Michel Foucaults orientierte Diskurstheorie. ${ }^{14}$ Sie stellt das zweite theoretisch-methodologische Standbein dieser Untersuchung dar, wobei die Zusammenführung beider Ansätze bereits in dem von Keller entwickelten Forschungsprogramm der Wissenssoziologischen Diskursanalyse ${ }^{15}$ verwirklicht worden ist. Der vorliegende Beitrag richtet sich im Wesentlichen nach diesem Programm. Auf eine Analyse der Sprecherpositionen folgt eine Analyse der Themen beziehungsweise Argumente der Debatte. Beide Analysen beruhen auf einem Korpus von 532 Texten (Pressemitteilungen, Reden, Blog-Einträgen, Interviews, Kampagnenmaterial), die im Rahmen ihrer Kampagnen von Seiten der identifizierten Wahlkampfteilnehmer veröffentlicht wurden. Als Zeitraum der Textproduktion wurde ein Intervall, beginnend mit dem Europäischen Rat im Juni 2009 und mit der Abstimmung am 2. Oktober 2009 endend, festgelegt. Die Texte wurden gelesen und unter Rückgriff auf die entsprechenden Verfahren qualitativer Datenanalyse kodiert. ${ }^{16}$ Überdies führte der Autor im Oktober 2009 Hintergrundinterviews mit den Sprechern von neun an der Debatte wesentlich beteiligten sozialen Akteuren.

\section{Sprecher und Sprecherpositionen im Lager der Ratifizierungsbefürworter}

Abbildung 1 veranschaulicht die identifizierten Sprecherpositionen in einer sogenannten Diskurslandkarte. Die Darstellung orientiert sich dabei an einem groben Links-RechtsSchema des politischen Spektrums. Die an der Referendumsdebatte beteiligten sozialen Akteure erscheinen auf dieser Karte also auf den ihnen zugewiesenen Plätzen, die mit Rahmen markiert sind, wobei ein breiter Rahmen bedeutet, dass es sich um eine Partei handelt, bei einem einfachen Rahmen ist es ein dauerhafter Interessenverband oder eine soziale Bewegung, bei gestrichelten Rahmen handelt es sich um eine Ad-hoc-Gruppe, vorübergehend und als Kampagnenorganisation ins Leben gerufen. Die Zahlenreihen sollen einen quantitativen Beleg für die Verteilung der bearbeiteten Texte auf die jeweiligen Akteure liefern. Dabei ist die erste Zahl die absolute Anzahl der Texte, die zweite, dritte und gegebenenfalls vierte Zahl enthalten deren relativen Anteil am Gesamt- beziehungsweise einem Teilkorpus.

12 Reiner Keller: Müll - die gesellschaftliche Konstruktion des Wertvollen. Die öffentliche Diskussion über Abfall in Deutschland und Frankreich, Opladen/Wiesbaden 1998, S. 33.

13 Vgl. als klassischen Text der Wissenssoziologie: Peter L. Berger/Thomas Luckmann: Die gesellschaftliche Konstruktion der Wirklichkeit. Eine Theorie der Wissenssoziologie, Frankfurt am Main 1969.

14 Vgl. vor allem Michel Foucault: Die Ordnung der Dinge: eine Archäologie der Humanwissenschaften, Frankfurt am Main 1974; Michel Foucault: Archäologie des Wissens, Frankfurt am Main 1981.

15 Reiner Keller: Wissenssoziologische Diskursanalyse. Grundlegung eines Forschungsprogramms, 2. Auflage, Wiesbaden 2008.

16 Vgl. Anselm L. Strauss: Grundlagen qualitativer Sozialforschung. Datenanalyse und Theoriebildung in der empirischen soziologischen Forschung, München 1998. 
Abbildung 1: Diskurslandkarte

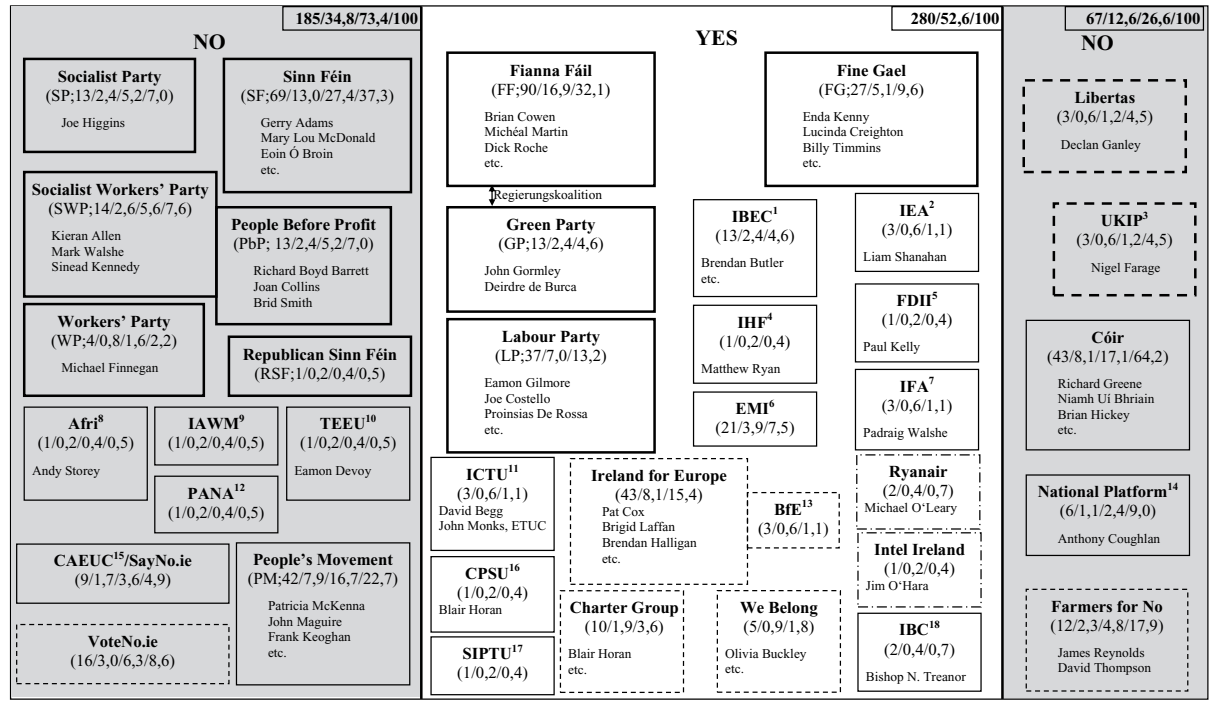

Anmerkungen: Für alle Zahlenreihen gilt: a/b/c/(d) a: absolute Anzahl der Texte/b: prozentualer Anteil von Gesamtkorpus/c: prozentualer Anteil von Teilkorpus ,Yes ' beziehungsweise ,No \%d: prozentualer Anteil von Teilkorpus ,No links “ beziehungsweise ,No rechts'. Eine vollständige Liste aller ausgewerteten Texte und Dokumente kann beim Autor erfragt werden (schuenemann@uni-landau.de). 1 Irish Business and Employers’ Confederation; 2. Irish Exporters Association; 3 UK Independence Party; 4 Irish Hotels Federation; 5 Food and Drink Industry Ireland; 6 European Movement Ireland; 7 Irish Farmers' Association; 8 Action from Ireland; 9 Irish Anti-War Movement; 10 Technical Engineering and Electrical Union; 11 Irish Congress of Trade Unions; 12 Peace and Neutrality Alliance; 13 Business for Europe; 14 National Platform EU Research \& Information Centre; 15 Campaign Against the EU Constitution; 16 Civil Public \& Services Union; 17 Services, Industrial, Professional and Technical Union; 18 Irish Bishops' Conference.

\section{Parteien}

Ähnlich wie vor dem Referendum 2008 haben sich auch 2009 mit Ausnahme von ,Sinn Féin“ alle im Parlament (Oireachtas) vertretenen Parteien für eine Ratifizierung ausgesprochen. Erstmals konnte auch die „Green Party“ als verbliebener kleiner Partner der Regierungskoalition mit „Fianna Fáil“ offiziell und aktiv für die Zustimmung zur Ratifizierung eines europäischen Vertrags werben, nachdem eine entsprechende Mitgliederbefragung am 18. Juli 2009 anders als im Vorjahr das innerparteilich vorgeschriebene Zweidrittelquorum erreicht hatte. ${ }^{17}$ Die Kampagne der Grünen 2009 war nur wenige Monate nach den Lokal- und Europawahlen vergleichsweise schlecht ausgestattet. Die Partei setzte aus eigenem Budget gerade einmal circa 5.000 Euro ein, erhielt zusätzlich allerdings eine ähnlich große Summe als Unterstützung von der „Europäischen Grünen Partei“. ${ }^{18}$ „We can’t tackle climate change alone“ war einer der zentralen Slogans der Kampagne. Neben der Sprecherin für europäische Angelegenheiten, der Senatorin Déirdre de Búrca, waren der Parteivorsitzende und amtierende Umweltminister John Gormley sowie der Energieminister Eamon Ryan weitere wichtige Sprecher der Kampagne.

17 Vgl. John Gormley: Greens back Lisbon Yes, 18.07.2009, abrufbar unter: http://www.greenparty.ie/en/news/ latest_news/greens_back_lisbon_yes (letzter Zugriff: 06.01.2010).

18 Diese und andere Informationen entstammen Mary Fitzgerald: Team Yes - who's who, how they are funded and what their strategy is, in: The Irish Times, 14.09.2009 sowie Interview des Autors mit der Sprecherin der „Green Party“ für europäische Angelegenheiten Deirdre de Búrca, 13.10.2009, Leinster House Dublin. 
Der große Partner in der Regierungskoalition, „Fianna Fáil“, ist als größte und in der republikanischen Geschichte mit Abstand am längsten regierende Partei des Landes bis heute für jeden europäischen Vertrag eingetreten, so auch in den Referenden 2008 und 2009. War die Partei im Juni 2008 aufgrund der gerade erst vollzogenen, mit dem Rücktritt des früheren Premierministers Bertie Ahern notwendig gewordenen Regierungsumbildung geschwächt gewesen, erging es ihr im Folgejahr kaum besser, im Gegenteil: Vor dem Hintergrund der verheerenden Wirtschaftskrise in Irland und angesichts unpopulärer Maßnahmen seitens der Regierung befand sich die Partei in einem weiteren Popularitätstief. So ging es für „Fianna Fáil“ im Referendum auch um das verbliebene Ansehen der Regierung und zahlreiche prominente Vertreter der Partei, allen voran Taoiseach Brian Cowen, Außenminister Micheál Martin als offizieller Leiter der Kampagne sowie Europaminister Dick Roche kämpften für die Zustimmung zum Vertragswerk. Mit etwas über 600.000 Euro lagen die veranschlagten Kosten für die nationale Partei dabei etwas unter dem Vorjahreswert. Davon kamen 125.000 Euro als Unterstützung von der „Fraktion der Allianz der Liberalen und Demokraten für Europa“ (ALDE), der die Abgeordneten von „Fianna Fáil“ im Europäischen Parlament angehören. ${ }^{19}$ Die zentrale Botschaft der Regierungspartei verdichtete sich in dem auf Tausende von Postern gedruckten Kernsatz „Ireland needs Europe“.

Die größte Oppositionspartei und aussichtsreiche zukünftige Regierungspartei, die konservative „Fine Gael“, leistete schon damit einen gewissen Beitrag zur erfolgreichen Abstimmung, dass sie sich öffentlich gegen die Umfunktionierung des Referendums in eine nationale Strafabstimmung zulasten der Koalition aus „Fianna Fáil“ und Grünen aussprach. Entsprechend sagte der Parteivorsitzende Enda Kenny in seiner Rede zum Start der Kampagne: "It would be very easy for me, as Leader of Fine Gael, to sit back and allow this referendum to be dominated by domestic political issues. While I fully understand people's anger, I do not want this crucial referendum to become a protest against the Government."20 Wie „Fianna Fáil“ hat auch „Fine Gael“ bisher jedes europapolitische Referendum seit 1972 unterstützt. Von den maximal 300.000 Euro, die für die Kampagne bereitgestellt wurden, kam ein knappes Drittel von der „Fraktion der Europäischen Volkspartei (Christdemokraten)“ (EVP), der die Europaabgeordneten von „Fine Gael“ angehören.

Die letzte Partei im Lager der Ratifizierungsbefürworter war die „Labour Party“, die ebenfalls für eine kommende Regierungsbildung bereitsteht. Wie bei den Grünen ist auch die Beziehung der „Labour Party“ zur Europäischen Union eher als ,critical engagement“ zu bezeichnen. Die Voraussetzungen für einen starken Wahlkampf für das ,Ja' waren keineswegs gut, hatte doch Umfragen zufolge eine Mehrheit von über 60 Prozent der Labour-Anhänger im Vorjahr gegen den Vertrag gestimmt. Unter dem Slogan „Work with Europe“ kämpften neben vielen anderen der Parteivorsitzende Eamon Gilmore, der Sprecher für europäische Angelegenheiten Joe Costello sowie die zwei Europaabgeordneten Alan Kelly und Proinsias De Rossa dennoch nach Kräften für die Ratifizierung. Die Kosten der Kampagne wurden mit circa 200.000 Euro veranschlagt, davon kam die Hälfte von der „Fraktion der Progressiven Allianz der Sozialisten und Demokraten“"21 (S\&D). ${ }^{22}$

19 Vgl. Fitzgerald: Team Yes - who's who, 2009 sowie Interview des Autors mit dem Minister of State for European Affairs Dick Roche, 15.10.2009, Department of the Taoiseach Dublin.

20 Enda Kenny: Kenny launches Fine Gael Lisbon Treaty Campaign, 03.09.2009, abrufbar unter: http://www.finegael.org/news/a/1013/article/ (letzter Zugriff: 14.10.2009).

21 Bis zur 6. Wahlperiode des Europäischen Parlaments firmierte die S\&D-Fraktion unter dem Namen ,Sozialdemokratische Fraktion im Europäischen Parlament".

22 Vgl. Fitzgerald: Team Yes - who's who, 2009 sowie Interview des Autors mit dem Sprecher der ,Labour Party“ für Europa Joe Costello, 07.10.2009, Leinster House Dublin. 


\section{Verbände und soziale Bewegungen}

Neben den Parteien warben auch zahlreiche Verbände und soziale Bewegungen für ein ,Ja' zum Vertrag von Lissabon. Starke Unterstützung fand die Ratifizierung insbesondere bei den Wirtschafts- und Industrieverbänden, allen voran dem irischen Arbeitgeberverband IBEC sowie der „Small Firms Association“ (SFA), der „Irish Exporters' Association“ und anderen Unternehmensverbänden. Ebenso sprach sich der 55 Einzelgewerkschaften umfassende „Irish Congress of Trade Unions“ (ICTU), darunter mitgliederstarke Gewerkschaften wie die „Civil Public \& Services Union“ (CPSU), die „Services, Industrial, Professional and Technical Union“ (SIPTU) etc., für die Ratifizierung aus. Darüber hinaus hatten sich führende Gewerkschaftsfunktionäre zur sogenannten „Charter Group“ zusammengetan, einer Ad-hoc-Gruppe, die in ihrer Kampagne insbesondere die Vorteile der mit dem LissabonVertrag (Art. 6 Abs. 1 Vertrag über die Europäische Union, EUV) für rechtsverbindlich erklärten Grundrechtecharta herausstellte. Das der europäischen Idee generell am stärksten verpflichtete „European Movement Ireland“ (EMI) musste, da aus öffentlichen Mitteln finanziert, in der zweiten Reihe bleiben - die Durchführung von Referenden ist spätestens seit dem ,McKenna'-Urteil des Supreme Court von 1995 stark reglementiert. Öffentliche Gelder dürfen seitdem nicht mehr für Kampagnen verwendet werden, darüber hinaus soll eine für jedes Referendum eingerichtete Referendum Commission für einen fairen Wahlkampf und unparteiische Information sorgen. Zuletzt mussten sich alle wahlkämpfenden Organisationen bei der SIPO-Agentur (Standards in Public Office) registrieren und ihre Wahlkampffinanzierung offenlegen. Vor diesem Hintergrund wurde scharf kritisiert, dass große Unternehmen wie „Intel Ireland“, „Microsoft“ und „Ryanair“ mit eigenen Kampagnen an der Debatte teilnehmen konnten, ohne entsprechende Transparenz über die Herkunft ihrer Mittel zu gewährleisten. „Intel Ireland“ und „Ryanair“ investierten jeweils circa 500.000 Euro in ihre Werbemaßnahmen zum Referendum.

Die größte und aktivste Ad-hoc-Gruppierung, die im Referendum 2009 für ein ,Ja“ kämpfte, war „Ireland for Europe“ (IfE) unter der Leitung von Pat Cox, einem früheren Europaabgeordneten für die mittlerweile aufgelösten liberalen „Progressive Democrats“. Cox war von 2002 bis 2004 Präsident des Europäischen Parlaments. Neben ihm wirkte als Vizedirektorin und Sprecherin von IfE Brigid Laffan, Wissenschaftlerin am „University College Dublin“ (UCD). IfE betonte die institutionelle Unabhängigkeit der Organisation und beschrieb sich entsprechend als Vertretung der Zivilgesellschaft. Dieser Ansatz war keineswegs neu. Auch in früheren Referenden hatten entsprechende Gruppen mehr oder weniger aktiv ins Wahlkampfgeschehen eingegriffen, zuletzt 2008 die „Irish Alliance for Europe“ unter der Führung des Labour-Abgeordneten Ruairi Quinn. Ohne Mandatsträger an der Spitze gelang die programmatische Distanzierung vom politischen Establishment IfE im Folgejahr allerdings glaubwürdiger. Entsprechend kämpferisch konnte Chief of Operations Andrew Byrne die eigene Ambition herausstreichen: "I'm not prepared to let politicians mess this up again. This is too important to leave to politicians."23

IfE wirkte auch als Generator für Satellitenorganisationen, die sich jeweils an einer regionalen, professionellen oder anderen gesellschaftlichen Zielgruppe orientierten. So entstanden „Sligo for Europe”, „Wicklow for Europe” etc., „Business for Europe”, „Lawyers for Europe” oder „Women for Europe”. Dem Netzwerk gelang es ferner, Prominente aus ver-

23 Ireland for Europe: Major Citizens' Group Promoting A 'Yes' Vote On Lisbon Launched, 21.06.2009, abrufbar unter: http://media.irelandforeurope.ie/?p=18 (letzter Zugriff: 25.11.2009). 
schiedenen Bereichen der Öffentlichkeit für seine Sache zu gewinnen. IfE finanzierte sich aus Spenden und veranschlagte eine Summe von maximal 500.000 Euro für seine Kampagne. ${ }^{24}$

Eine ähnliche zivilgesellschaftliche Zusammensetzung wie IfE kennzeichnete die zwar unabhängige, aber mit IfE die Büroräume teilende Gruppe „Generation Yes“. Die Bewegung zielte vor allen Dingen auf junge Wähler, die als Problemgruppe in der Vorjahresabstimmung ausgemacht worden waren. Zuletzt folgte die Organisation „We Belong“ unter der Leitung von Olivia Buckley einem ganz ähnlichen Ansatz, sie band ebenfalls verschiedene populäre Figuren aus Wirtschaft und Gesellschaft in die Kampagne ein.

\section{Sprecher und Sprecherpositionen im Lager der Ratifizierungsgegner}

\section{Ratifizierungsgegner auf der politischen Rechten}

Die größte Veränderung in den Sprecherkonstellationen zwischen den Referenden 2008 und 2009 war sicher auf der Seite der Ratifizierungsgegner zu verzeichnen. „Libertas“, die als Think-Tank gegründete EU-kritische Organisation unter der Führung des Unternehmers Declan Ganley, hatte 2008 einen großen Anteil am Erfolg für das ,Nein'-Lager gehabt. 2009 hingegen war „Libertas“ zunächst nicht präsent und Ganley gab vor, sich nicht erneut an der Debatte beteiligen zu wollen. Gut zwei Wochen vor der eigentlichen Abstimmung, Mitte September, stieg Ganley jedoch wieder mit seinen Argumenten sowie einer Libertas-Kampagne gegen die Ratifizierung in den Wahlkampf ein, die allerdings längst nicht die gleichen Ausmaße hatte wie im Vorjahr. ${ }^{25}$ Zum politischen Hintergrund von „Libertas“ gehörte 2009 auch der weitgehend gescheiterte Versuch, sich auf europäischer Ebene als Partei zu etablieren (daher der gestrichelte breite Rahmen in Abbildung 1). Bei den Europawahlen im Juni 2009 trat „Libertas“ in 14 EU-Mitgliedstaaten mit Wahllisten an, gewann jedoch nur einen einzigen Sitz im Parlament. ${ }^{26}$

Die größte flächendeckend aktive Organisation auf der politischen Rechten war in beiden Referenden die souveränistische Organisation „Cóir“. Ihre Zuordnung im groben LinksRechts-Schema rechtfertigt sich anders als bei „Libertas“ nicht anhand einer soziökonomischen, sondern einer sozialethischen Konfliktlinie. „Cóir“" wurde von den Ratifizierungsbefürwortern und vielen anderen Beobachtern der Debatte mit der fundamental-religiösen ProLife-Organisation „Youth Defence“ assoziiert. Auch wenn sich die Vertreter von „Cóir“, allen voran ihr sichtbarster Sprecher, der referendumserfahrene Aktivist Richard Greene, gegen diese Assoziation zur Wehr setzten, kam sie durchaus nicht von ungefähr. Die beiden Organisationen teilten sich ihr Hauptquartier in der Dubliner Capel Street und auch einen beträchtlichen Teil seiner Wahlkämpfer auf den Straßen bezog "Cóir“ von „Youth Defence“. ${ }^{27}$ Zudem besteht eine inhaltliche Gemeinsamkeit darin, dass „Youth Defence“ einen dauerhaften Abwehrkampf gegen die Einführung der legalen Abtreibung in Irland führt und auch „Cóir“ tat sich insbesondere 2008, in etwas geringerem Ausmaß aber auch 2009 mit

24 Vgl. Fitzgerald: Team Yes - who's who, 2009 sowie Interview des Autors mit Ireland for Europe Vice Director Brigid Laffan, 05.10.2009, University College Dublin.

25 Vgl. Declan Ganley: EU-Vertrag: "Die Iren werden wieder Nein sagen“, in: Die Presse, 14.09.2009 sowie Fitzgerald: Team No - who's who, how they are funded and their strategy, in: The Irish Times, 15.09.2009.

26 Diesen Sitz erreichte „Libertas“ in Frankreich auf einer gemeinsamen Liste mit der ohnehin bei Europawahlen traditionell erfolgreichen rechtskonservativen Partei „Mouvement pour la France“ (MPF) um Philippe de Villiers.

27 Vgl. Fitzgerald: Team No - who's who, 2009 sowie Interview des Autors mit Cóir Spokesperson Richard Greene, 16.10.2009, Montrose Hotel Dublin. 
ausgesprochen wirksamer sozialethisch motivierter Kritik am Lissabon-Vertrag hervor, der angeblich die Legalisierung von Abtreibung, Sterbehilfe, gleichgeschlechtlicher Ehe und Prostitution befördere. Auch im zweiten Lissabon-Referendum gelang es „Cóir“ wieder, die Debatte mit einem in extremer Weise formulierten Thema zu dominieren: der angeblichen Bedrohung des irischen Mindestlohns (siehe unten).

Ein beliebter Redner auf Wahlkampfveranstaltungen von „Cóir“ war der in Irland bereits langjährig aktive EU-kritische Wissenschaftler Anthony Coughlan. Er leitet als Direktor die Organisation „National Platform EU Research \& Information Centre“, die seit den achtziger Jahren für ein Europa unabhängiger Nationen eintritt. Coughlan und die „National Platform“

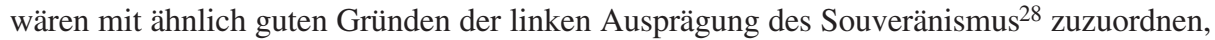
werden hier allerdings aufgrund der ebenfalls formulierten sozialethischen Bedenken auf der politischen Rechten verortet. ${ }^{29}$

Auf dieser finden sich schließlich die landwirtschaftliche Ad-hoc-Gruppierung „Farmers for No“ sowie die britische „UK Independence Party“ (UKIP).${ }^{30}$ Die beiden Organisationen vereint, dass sie als einzige der untersuchten sozialen Akteure mit einem drohenden EU-Beitritt der Türkei gegen die Ratifizierung des Lissabon-Vertrags argumentierten. Die UKIP unter der Führung des Europaabgeordneten Nigel Farage, war in der irischen Öffentlichkeit freilich leicht zu diskreditieren. Einmischungen von der Nachbarinsel werden nicht gern gesehen und wurden dementsprechend nicht nur von den Befürwortern der Ratifizierung zum Teil scharf kritisiert.

\section{Ratifizierungsgegner auf der politischen Linken}

Die einzige gegen die Ratifizierung des Vertrags kämpfende im Oireachtas vertretene Partei war die linksrepublikanische „Sinn Féin“. Sie geht in ihrer heutigen Form aus dem NordirlandKonflikt hervor. Als ihr Alleinstellungsmerkmal versteht sie ihre politische Präsenz auf der gesamten irischen Insel. Die europapolitische Ausrichtung der Partei ist eher kritisches Engagement denn Fundamentalverweigerung. In beiden Referenden warb „Sinn Féin“ mit der Kennzeichnung des Lissabon-Vertrags als „,bad deal“ und forderte die Regierung auf, einen besseren Vertrag auszuhandeln. 2009 wurde dieses Argument um die lautstarke Kritik an der Wiedervorlage desselben Dokuments ergänzt: „Same Treaty, Same Answer“. 31 An vorderster Front stand wie auch schon 2008 die Vizepräsidentin der Partei Mary Lou McDonald. Anders als im Vorjahr war McDonald 2009 nicht mehr Inhaberin eines Sitzes im Europäischen Parlament für ihren Dubliner Wahlkreis; diesen hatte sie bei den vorangegangenen Wahlen im Juni 2009 verloren. Die Partei veranschlagte Ausgaben für ihre Kampagne von circa 100.000 Euro, einschließlich finanzieller Unterstützung in Höhe von 15.000 Euro seitens der „Konföderalen Fraktion der Vereinigten Europäischen Linken/Nordischen Grünen Linken“ (KVEL/NGL), der die verbliebene nordirische Sinn Féin-Abgeordnete im Europäischen Parlament angehört. ${ }^{32}$

28 Vgl. zum Begriff Souveränismus (französisch souverainisme) Pierre Martin: Comment analyser les changements dans les systèmes partisans d'Europe occidentale depuis 1945, in: Revue internationale de politique comparée 2/2007, S. 263-280; siehe ferner zur Konzeption einer entsprechenden europapolitischen Konfliktlinie zwischen Integrationismus und Souveränismus Simon Hix/Christopher Lord: Political parties in the European Union, Basingstoke u.a. 1997.

29 Hier zeigt sich ein weiteres Mal die analytische Schwierigkeit, dem politischen System Irlands und der irischen Gesellschaft mit einem Links-Rechts-Schema beizukommen.

30 UKIP ist keine irische Partei und daher mit einem gestrichelten breiten Rahmen in Abbildung 1 dargestellt.

31 Vgl. zum Beispiel Caoimhghín Ó Caoláin: Same Treaty, Same Answer - Ó Caoláin, 17.09.2009, abrufbar unter: http://www.sinnfein.ie/contents/17368 (letzter Zugriff: 08.10.2009).

32 Vgl. Fitzgerald: Team No - who's who, 2009 sowie Interview des Autors mit Sinn Féin Director of strategy Eoin Ó Broin, 10.10.2009, Sinn Féin Headquarters Dublin. 
Ebenfalls ausgesprochen aktiv insbesondere während des zweiten Lissabon-Referendums war der erst im Juni 2009 ins Parlament eingezogene Europaabgeordnete Joe Higgins. Die von ihm geführte „Socialist Party“ ging Anfang der 1990er Jahre als marxistische Abspaltung aus der „Labour Party“ hervor. Die „Socialist Party“ investierte ein Budget von circa 60.000 Euro, wobei ein Großteil ebenfalls von der KVEL/NGL stammte.

Neben diesen beiden Parteien kämpften noch andere linke Splitterparteien mit ähnlichen Argumenten für ein ,Nein“ zum Lissabon-Vertrag wie zum Beispiel die „Socialist Workers' Party“ (SWP), deren Wahlkandidaten mitunter auf gemeinsamen Wahllisten mit denjenigen der ebenfalls in der Debatte aktiven „People Before Profit Alliance“ antreten, daneben die „Workers' Party“, „Éirígi“" sowie „Republican Sinn Féin“ (RSF). Außerdem waren mit dem „Irish Anti-War Movement“, der „Peace \& Neutrality Alliance“(PANA) sowie „Action from Ireland“(Afri) auch drei Gruppen aus der irischen Friedensbewegung aktiv an der linken Kampagne gegen den Lissabon-Vertrag beteiligt und formulierten jeweils vornehmlich militarisierungskritische Argumente.

Eine Sonderrolle im linken Lager der Ratifizierungsgegner nimmt das „People's Movement“ (PM) ein, das mit der Debatte um den Verfassungsvertrag als Ad-hoc-Gruppe entstanden ist, sich seitdem allerdings flächendeckend institutionalisiert und in beiden Lissabon-Referenden aktiv gegen den Vertrag gekämpft hat. Das PM definiert sein Anliegen vornehmlich als Bewahrung der Volkssouveränität gegenüber einer weiteren Entwicklung der Europäischen Union in einen undemokratischen Superstaat. Das erste Gesicht der Kampagne war wieder einmal die bekannte EU-Skeptikerin und ehemalige Europaabgeordnete der „Green Party“ Patricia McKenna. Sie war 2009 aus der Partei ausgetreten und hatte erfolglos versucht ihren Parlamentssitz als Unabhängige zu verteidigen. Das PM finanzierte seine circa 20.000 Euro teure Kampagne ausschließlich über private Spenden. ${ }^{33}$

Alle bisher genannten Gruppen im linken ,Nein'-Lager außer Afri und RSF kooperierten in der schon 2005 gegründeten übergreifenden Initiative „Campaign Against the EU Constitution“ (CAEUC), die während der Referenden auch unter dem Titel „No to Lisbon“ (,sayno.ie“) auftrat. Die CAEUC steht über den irischen Kontext hinaus in Verbindung mit den ATTAC-Gruppierungen in Frankreich und Österreich. Eine ähnliche parteiübergreifende Kampagne auf der Linken war „VoteNo.ie“, gegründet vom SWP-Aktivisten Kieran Allen. Zuletzt waren im linken ,Nein'-Lager auch zwei Gewerkschaften zu finden, nämlich zum einen die „Technical Engineering and Electrical Union“ (TEEU) sowie die mitgliederstarke, aber zum größten Teil britische Gewerkschaft „Unite“.

\section{Themen und Argumente in der Ratifizierungsdebatte}

Während der mehrmonatigen Referendumsdebatte wurden von den Akteuren auf beiden Seiten so viele politisch-gesellschaftliche Themen angesprochen, dass sie hier nicht alle ausführlich behandelt werden können. Vielmehr ergibt eine quantitative Auswertung der bearbeiteten und kodierten Texte eine Häufigkeitsverteilung, sodass ersichtlich ist, in wie vielen der bearbeiteten Texte ein Argument vorkommt. Für beide Lager sind jeweils zunächst die fünf Argumente/Kodes ausgewählt worden, die in einer hohen Anzahl von Texten enthalten sind. Sie sind mit den jeweiligen Häufigkeiten in den Tabellen 1 und 2 aufgeführt. Die jeweils dritte Spalte enthält die Kürzel der sozialen Akteure, die sich innerhalb des bearbeiteten Textkorpus dieser Argumente bedienen. Jeweils die drei häufigsten Argumente der beiden Seiten sollen ausführlicher vorgestellt werden. Der Rückgriff auf die Häufigkeitsverteilung erlaubt dabei letztlich nur Aussagen über die quantitative Bedeutung eines Arguments im Wahlkampf, nicht über seine tatsächliche Bedeutung für den Wahlausgang.

33 Vgl. Interview des Autors mit People's Movement Secretary Frank Keoghan, 13.10.2009, Dublin. 


\section{Ratifizierungsgegner}

\section{Wiederwahlkritik}

Das im Rahmen dieser Untersuchung als Wiederwahlkritik bezeichnete Argument ist in den vorangegangenen Abschnitten bereits gelegentlich angeklungen. Es spielte eine große Rolle für das Referendum und kam in über 40 Prozent der bearbeiteten Texte des, Nein'-Lagers vor. Die Wiederwahlkritik lag im Grunde seit dem gescheiterten ersten Referendum in der Luft und entsprechend kritisch wurde jeder Schritt der Regierung auf europäischer Ebene beobachtet. Später sträubten sich die Ratifizierungsgegner in der Regel, die von der Regierung erreichten Vereinbarungen der Staats- und Regierungschefs als Garantien zu bezeichnen, so wie es die ,Ja'-Seite tat. Sie sprachen dagegen bestenfalls von ,so called , guarantees““, eher noch von ,assurances“ oder „,clarifications“.34 Auf jeden Fall wurden die Vereinbarungen für vollkommen wertlos befunden. ${ }^{35}$ Aus Sicht nahezu aller Ratifizierungsgegner auf beiden Seiten des politischen Spektrums hatte sich der Abstimmungsgegenstand nicht im Geringsten verändert, weshalb auch die Antwort nur dieselbe sein könnte wie im Jahr zuvor: „As it is exactly the same treaty, Sinn Féin is urging people to give it the same answer and vote No on 2 October." ${ }^{36}$ Die sogenannten Garantien der Regierung waren also über den gesamten Verlauf der Debatte selbst Gegenstand intensiver Deutungskämpfe, was sich auch an der Gewichtung des entsprechenden Abwehrarguments gegen die Wiederwahlkritik unter den Argumenten der, Ja'-Seite ablesen lässt (siehe Tabelle 2).

\section{Tabelle 1: Die häufigsten Argumente der Ratifizierungsgegner}

\begin{tabular}{|l|l|l|}
\hline Bezeichnung des Arguments & $\begin{array}{l}\text { Anzahl/An- } \\
\text { teil der Texte }^{\mathbf{a}}\end{array}$ & soziale Akteure \\
\hline Neoliberalismuskritik & $127 / 23,9 / 50,4$ & $\begin{array}{l}\text { SF, SP, PbP, SWP, WP, RSF, Pana, Afri, PM, CA- } \\
\text { EUC, VoteNo, TEEU, Cóir, NP }\end{array}$ \\
\hline Wiederwahlkritik & $107 / 20,1 / 42,5$ & $\begin{array}{l}\text { SF, SP, PbP, Lib., SWP, NP, WP, VoteNo, AntiWar, } \\
\text { CAEUC, Cóir, Pana, PM, RSF, UKIP }\end{array}$ \\
\hline Souveränitätsargument & $86 / 16,2 / 30,7$ & $\begin{array}{l}\text { SF, SP, PbP, Lib., NP, WP, VoteNo, SWP, AntiWar, } \\
\text { CAEUC, Cóir, FfNo, Pana, PM, RSF, UKIP }\end{array}$ \\
\hline $\begin{array}{l}\text { Plan B-Argument/ } \\
\text { GA Zukunft }\end{array}$ & $69 / 13 / 27,4$ & $\begin{array}{l}\text { SF, SP, PbP, Lib., NP, WP, VoteNo, TEEU, SWP, } \\
\text { SIPTU, CAEUC, Cóir, FfNo, Pana, PM, RSF, UKIP }\end{array}$ \\
\hline Militarisierungskritik & $63 / 11,8 / 25$ & $\begin{array}{l}\text { SF, SP, PbP, NP, VoteNo, SWP, Afri, AntiWar, CA- } \\
\text { EUC, Cóir, Pana, PM, RSF }\end{array}$ \\
\hline
\end{tabular}

a: Anzahl/Anteil aller Texte in Prozent/Anteil der Texte des jeweiligen Lagers in Prozent; GA: Gegenargument; SF: Sinn Féin; SP: Socialist Party; PbP: People before Profit; SWP: Socialist Workers' Party; WP: Workers' Party; RSF: Republican Sinn Féin; Pana: Peace \& Neutrality Alliance; Afri: Action from Ireland; PM: People's Movement; CAEUC: Campaign Against the EU Constitution; TEEU: Technical Engineering and Electrical Union, NP: National Platform EU Research \& Information Centre; Lib.: Libertas; AntiWar: Irish Anti-War Movement; FfNo: Farmers for No; UKIP: UK Independence Party.

34 Vgl. zum Beispiel Dana R. Scallon: Dana Urges NO vote, 30.09.2009, abrufbar unter: http://www.coircampaign.org/index.php/news-articles/archives/232-dana-urges-no-vote (letzter Zugriff: 09.06.2010) beziehungsweise Patricia McKenna: Government's claim that pressure was put on EU to accommodate Ireland is utter nonsense, says McKenna, 08.07.2009, abrufbar unter: http://www.people.ie/press/090708.pdf (letzter Zugriff: 13.10.2009).

35 Vgl. zum Beispiel UKIP: Still chance of upset in Ireland, 22.09.2009, abrufbar unter: http://www.ukip.org/ content/latest-news/1255-still-chance-of-upset-in-ireland (letzter Zugriff: 28.12.2009).

36 Sinn Féin: Sinn Féin unveils 'No to Lisbon' poster campaign, in: An Phoblacht, 17.09.2009. 


\section{Souveränitätsargument}

Das grundlegendste Anliegen für eine ganze Reihe der beteiligten Akteure war die Bewahrung der nationalen Souveränität Irlands gegenüber einer angeblich immer weiter in staatliche Hoheitsrechte eingreifenden Europäischen Union. Das Souveränitätsargument hat viele Facetten, die von Autarkievorstellungen auf der Rechten über Vorstellungen von Volkssouveränität bis hin zu Erwartungen wirtschaftspolitischer Steuerungsfähigkeit auf der Linken reichen. Die verschiedenen Aspekte des Souveränitätsarguments berührten eine Vielzahl konkreter Neuerungen des Lissabon-Vertrags. Anstoß wurde vor allem am verstärkten Einsatz qualifizierter Mehrheitsentscheidungen genommen. Dabei würde überdies nach den Regeln der mit dem Vertrag eingeführten doppelten Mehrheit das Stimmengewicht Irlands drastisch reduziert, während die entsprechenden Gewichte der größten Mitgliedstaaten erheblich wüchsen. So erklärte beispielsweise Anthony Coughlan mit Bezug auf Art. 16 Abs. 4 EUV: „By basing EU law-making primarily on population size, the Lisbon Treaty would double Germany's relative voting strength on the EU Council of Ministers [...] while halving Ireland's vote from $2 \%$ to $0.8 \%$." 37

Ferner wurde kritisiert, dass das Vertragswerk durch die enthaltenen Flexibilitätsklauseln sowie das vereinfachte Änderungsverfahren nach Art. 48 EUV zu einer Art ,self-amending treaty" würde und Referenden so in Zukunft gar gänzlich würden umgangen werden könnten. Zuletzt verwandle die Europäische Union sich mit dem Vertrag von Lissabon endgültig in einen undemokratischen Superstaat. ${ }^{38}$

\section{Neoliberalismuskritik}

Eine in vielen europäischen Gesellschaften insbesondere auf der politischen Linken verbreitete Kritik an der europäischen Integration zielt auf die angeblich neoliberale Ausrichtung der Europäischen Union. Mit Blick auf die Inhalte des Lissabon-Vertrags wurde die in einem übergeordneten Kode zusammengefasste Neoliberalismuskritik auch im irischen Referendum 2009 von zahlreichen Akteuren innerhalb des ,Nein'-Lagers auf beiden Seiten des politischen Spektrums (mit Ausnahme von „Libertas“ und UKIP) aktualisiert. Sie erreicht in der quantitativen Auswertung der vorgenommenen Kodierungen den höchsten Rang unter den ,Nein'-Argumenten. Entsprechende Fundstellen wurden in knapp über 50 Prozent der bearbeiteten Texte des ,Nein'-Lagers identifiziert.

Die Neoliberalismuskritik berührt als allgemeiner Ausdruck des sozioökonomisch motivierten Unbehagens über die Entwicklung der europäischen Integration eine Vielzahl von Aspekten, die im Rahmen der Untersuchung mit unterschiedlichen Subkodes ausdifferenziert wurden. Die wichtigsten unter ihnen sind die angeblich durch die Europäische Union und den Lissabon-Vertrag beförderte Gefährdung des öffentlichen Dienstes sowie der Sozialabbau. In Bezug auf letzteren diente insbesondere der Europäische Gerichtshof aufgrund einiger als arbeitnehmerfeindlich bewerteten Urteile der vergangenen Jahre (,Laval', ,Viking', ,Rüffert', ,Luxemburg') als beliebtes Feindbild. ${ }^{39}$ Die Kritik an der nicht zuletzt durch die Urteile des Europäischen Gerichtshofes beförderten Lohnkonkurrenz im freien Markt der Arbeitskräfte gipfelte in der Warnung von „Cóir“, der irische Mindestlohn von

37 Anthony Coughlan: Explanatory document on Lisbon, 09.07.2009, abrufbar unter: http://www.nationalplatform.org/2009/07/09/explanatory-document-on-lisbon/ (letzter Zugriff: 04.12.2009).

38 Vgl. People before Profit: Ten Reasons to Vote NO, 01.10.2009, abrufbar unter: http://www.people-beforeprofit.org/node/220 (letzter Zugriff: 20.11.2009).

39 Als Überblick zu dieser Debatte siehe: Franz C. Mayer: Der EuGH als Feind? Die Debatte um das soziale Europa in der europäischen Rechtsprechung, in: integration 3/2009, S. 246-265. 
derzeit über acht Euro drohe sich mit einer Ratifizierung des Lissabon-Vertrags mittels Lohnkonkurrenz auf 1,84 Euro zuzubewegen: ,€ $1.84=$ the average minimum wage of the EU accession states. If Lisbon passes those rates can apply to foreign workers contracted here." ${ }^{40}$ Aktuellen Bezug nahmen die Kritiker auch auf die Wirtschafts- und Finanzkrise, die von derselben neoliberalen Politik ausgelöst worden sei, die nun durch den Vertrag von Lissabon festgeschrieben werden solle. Dies kann als Versuch der Ratifizierungsgegner angesehen werden, dem in der tiefen Wirtschaftskrise potenziell besonders wirksamen ökonomischen Argument des ,Ja'-Lagers direkt zu widersprechen.

\section{Weitere Argumente und Zwischenfazit}

Im Jahr 2008 hatte das ,Nein“-Lager mit der Kritik am Informationsmangel und dem plakativen Slogan ,if you don't know vote no“ viele Stimmen gewinnen können. In der Referendumsdebatte 2009 nach anderthalb Jahren Beschäftigung mit dem Vertragswerk tauchte das Argument hingegen verständlicherweise kaum noch auf. Andere Argumente von 2008 wie die Militarisierungskritik (siehe Tabelle 1) mit der Betonung außenpolitischer Neutralität, die sozialethisch motivierte Kritik (Abtreibung, Sterbehilfe etc.) sowie die Warnung vor einer möglichen Harmonisierung der Unternehmenssteuern wurden trotz der Klarstellungen auf EU-Ebene erneut von den jeweiligen Akteuren vorgebracht. Dominante Themen der Ratifizierungsgegner waren das Souveränitätsargument, die Wiederwahlkritik sowie die Neoliberalismuskritik mit besonderem Schwerpunkt auf der Warnung vor Sozialabbau und Lohndumping im Binnenmarkt.

\section{Ratifizierungsbefürworter}

\section{Zukunft in Europa/Katastrophenargument}

Das in den meisten Texten der ,Ja'-Seite identifizierte Argument (48,9 Prozent) war eines, das sich nicht auf konkrete Vertragsinhalte bezog, sondern mit dem die Ratifizierungsbefürworter vielmehr vor den negativen Auswirkungen einer neuerlichen Ablehnung des Vertragswerks auf internationaler, insbesondere europäischer Ebene warnten. Irland würde sich mit einem zweiten ,Nein“ innerhalb Europas isolieren und sich damit aus der politisch einflussreichen Kerngruppe europäischer Staaten entfernen. Eine weitere Ablehnung des Vertragswerks würde die Wahrnehmung Irlands im Ausland stark beschädigen. Auf der anderen Seite wäre ein ,Ja‘ im zweiten Anlauf ein angebrachtes Zeichen der Solidarität gegenüber den europäischen Partnern, die im Rahmen der vereinbarten Garantien so weit auf die irische Bevölkerung zugegangen seien. Die Aufforderung an die Bevölkerung, mit einem positiven Votum Irlands Stellung ,at the heart of Europe“ zu bewahren, wurde von nahezu allen Akteuren im ,Ja'-Lager in ebendiesem Wortlaut formuliert, so auch von Europaminister Dick Roche: ,by voting YES we the Irish people will determine that our nation stays at the heart of [the] European Union. If we vote YES we send out a powerful message about Ireland and Europe."41 In seiner häufigsten Verbindung mit dem Wirtschaftsargument (siehe unten) war das Zukunfts- oder je nach Stoßrichtung auch Katastrophenargument dazu geeig-

40 Cóir: Club Lisbon, 2009, abrufbar unter: http://www.coircampaign.org/images/LeafletsPosters/leaf_club\%2 Olisbon.pdf (letzter Zugriff: 09.06.2010).

41 Dick Roche: Speech by Dick Roche TD, Minister for European Affairs, 09.07.2009, abrufbar unter: http:// www.fiannafail.ie/news/entry/speech-by-dick-roche-td-minister-for-european-affairs/ (letzter Zugriff: 14.10.2009). 
net, die sozioökonomische Verunsicherung der Bevölkerung vor dem Hintergrund der verheerenden Wirtschaftskrise anzusprechen.

Tabelle 2: Die häufigsten Argumente der Ratifizierungsbefürworter

\begin{tabular}{|l|l|l|}
\hline Bezeichnung des Arguments & $\begin{array}{l}\text { Anzahl/An- } \\
\text { teil der Texte }^{\mathbf{a}}\end{array}$ & soziale Akteure \\
\hline $\begin{array}{l}\text { Zukunft in Europa/ } \\
\text { Katastrophenargument }\end{array}$ & $137 / 25,8 / 48,9$ & $\begin{array}{l}\text { FF, FG, GP, LP, Intel, WB, SIPTU, Ryan, IHF, BfE, } \\
\text { Bish, CG, ICTU, FDII, IBEC, IEA, IFA, IfE }\end{array}$ \\
\hline Wirtschaftsargument & $134 / 25,2 / 47,9$ & $\begin{array}{l}\text { FF, FG, GP, LP, Intel, WB, Ryan, IHF, BfE, Bish, CG, } \\
\text { ICTU, FDII, IBEC, IEA, IFA, IfE }\end{array}$ \\
\hline $\begin{array}{l}\text { GA Wiederwahlkritik/ } \\
\text { Garantien }\end{array}$ & $92 / 17,3 / 32,9$ & $\begin{array}{l}\text { FF, FG, GP, LP, SIPTU, BfE, Bish, CG, IBEC, IfE, } \\
\text { EMI }\end{array}$ \\
\hline $\begin{array}{l}\text { Irland in Europa/ } \\
\text { Celtic Tiger }\end{array}$ & $89 / 16,7 / 31,8$ & $\begin{array}{l}\text { FF, FG, GP, LP, Intel, WB, Ryan, BfE, CG, ICTU, } \\
\text { IBEC, IEA, IFA }\end{array}$ \\
\hline $\begin{array}{l}\text { Effizienz/Effektivität/ } \\
\text { Transparenz }\end{array}$ & $77 / 14,5 / 27,5$ & FF, FG, GP, LP, IHF, BfE, CG, IBEC, IfE, EMI \\
\hline
\end{tabular}

a: Anzahl/Anteil aller Texte in Prozent/Anteil der Texte des jeweiligen Lagers in Prozent; GA: Gegenargument; FF: Fianna Fáil; FG: Fine Gael; GP: Green Party; LP: Labour Party; WB: We Belong; SIPTU: Services, Industrial, Professional and Technical Union; Ryan: Ryanair; IHF: Irish Hotels Federation; BfE; Business for Europe; Bish: Irish Bishops' Conference; CG: Charter Group; ICTU: Irish Congress of Trade Unions; FDII: Food and Drink Industry Ireland; IBEC: Irish Business and Employers' Confederation; IEA: Irish Exporters Association; IFA: Irish Farmers' Association; IfE: Ireland for Europe; EMI: European Movement Ireland.

\section{Irland in Europa/Keltischer Tiger}

Die mit ,Irland in Europa/Keltischer Tiger" kodierten Textstellen haben in der Regel die Form von übergeordneten Erzählungen innerhalb der Referendumsdebatte. Sie erzählen die Erfolgsgeschichte von Irlands wirtschaftlichem Aufstieg seit Eintritt in die Europäische Gemeinschaft und insbesondere während der jüngeren Boomperiode des Keltischen Tigers. Sie berichten von der Entwicklung der irischen Exportwirtschaft dank des europäischen Binnenmarkts, betonen die Bedeutung desselben im Hinblick auf die Attraktivität Irlands für ausländische (insbesondere US-amerikanische) Direktinvestitionen und verweisen nicht zuletzt auf die umfangreiche Förderung der irischen Landwirtschaft sowie öffentliche Infrastrukturund Ausbildungsmaßnahmen durch die entsprechenden EU-Fonds. So heißt es zum Beispiel in der Rede des Fine Gael-Vorsitzenden Enda Kenny zum Start der Kampagne: „I firmly believe that EU membership has been pivotal to Ireland's success story.“42 Darüber hinaus wird hier auch mit dem politischen Einflusszuwachs Irlands während der über 30-jährigen Mitgliedschaft in der Europäischen Union beziehungweise zurvor der Europäischen Gemeinschaft argumentiert.

\section{Wirtschaftsargument}

Die größte Kontextveränderung zwischen den Referenden 2008 und 2009 wurde durch die Wirtschafts- und Finanzkrise ausgelöst, die Irland härter getroffen hat als die meisten anderen EU-Mitgliedstaaten. Die Ära des Keltischen Tigers war im Jahr 2008 spätestens beendet und Irland rutschte in eine Rezession. Innerhalb von nur sechzehn Monaten stieg die Ar-

42 Enda Kenny: Kenny launches Fine Gael Lisbon Treaty Campaign, 03.09.2009, abrufbar unter: http://www.finegael.org/news/a/1013/article/ (letzter Zugriff: 14.10.2009). 
beitslosigkeit von 6,3 Prozent (Juni 2008) auf 12,3 Prozent (Oktober 2009). ${ }^{43}$ Wegen geringerer Steuereinnahmen und der daraus folgenden höheren Neuverschuldung verstieß Irland 2008 erstmals gegen die Auflagen des Stabilitäts- und Wachstumspakts. Die Konsolidierung der öffentlichen Finanzen ist mit umfassenden Stellenstreichungen im öffentlichen Dienst und Kürzungen der Sozialleistungen verbunden gewesen. ${ }^{44}$

Vor diesem düsteren Hintergrund und angesichts der großen Verunsicherung in der Bevölkerung setzten die Ratifizierungsbefürworter 2009 stark auf wirtschaftliche Themen. Die unter dem Wirtschaftsargument rubrizierten Aussagen fanden sich in knapp 48 Prozent der bearbeiteten Texte des ,Ja'-Diskurses. Natürlich wurden unter dieser breiten Kategorie unterschiedliche Aspekte angesprochen, die anhand von Subkodes überprüft wurden. Dabei standen zwei Aussagen im Vordergrund. Zum Ersten wurde auf die Abhängigkeit Irlands von ausländischen Direktinvestitionen verwiesen, die es unbedingt erforderlich mache, dass Irland eine starke Stellung innerhalb der Europäischen Union behalte (siehe Zukunftsargument). ${ }^{45}$

Häufiger noch wurde eine Zustimmung zum Lissabon-Vertrag allgemein als erster Schritt auf dem Weg zur wirtschaftlichen Erholung, zum Aufschwung dargestellt. „Ireland for Europe" verdichtete diese Aussage am stärksten zum Bild des Scheidewegs zwischen „ruin“ versus „recovery“ und druckte eine entsprechend beschilderte Weggabelung auf Tausende von Postern. Auch in ihren sechs Gründen für ein ,Ja ' wurde die Wegmetapher eingesetzt: „To choose the path to recovery and avoid the road to ruin.”46 „Path“ und ,road to recovery" gehörten zu den häufigsten Formeln im Diskurs der Ratifizierungsbefürworter. Dass sich die Europäische Union insbesondere durch die Unterstützung irischer Banken seitens der Europäischen Zentralbank seit Beginn der Krise bereits als Rettungsanker erwiesen hatte, kam im folgenden immer wieder zitierten Wortspiel zum Ausdruck: „It has become a cliché to suggest that the only difference between Ireland and Iceland is one letter. Actually the difference is two letters: $\mathrm{E}$ and $\mathrm{U} .{ }^{\text {“47 }}$

\section{Weitere Argumente des , Ja'-Lagers}

Das Wirtschaftsargument und darin der Verweis auf die Wirtschaftskrise waren das Kernthema für die Ratifizierungsbefürworter 2009. Das Zukunftsargument sowie die Erzählung vom Keltischen Tiger könnte man so auch als narrative Rahmung des zentralen ökonomischen Arguments deuten. Auffallend ist, dass der ganze Komplex sich nicht konkret auf Bestimmungen des Lissabon-Vertrags bezog, was zu heftiger Kritik bei den Ratifizierungsgegnern führte. Doch hatte auch die ,Ja'-Seite wichtige, wenn auch weniger häufige Argumente mit konkretem Vertragsbezug vorzubringen, so das Argument für institutionelle Reformen (Effizienz, Effektivität, Transparenz; siehe Tabelle 2), das Charta-Argument, das die

43 Beide Zahlen von Eurostat: Datenbank Euro-Indikatoren - Harmonisierte Arbeitslosigkeitsrate, abrufbar unter: http://epp.eurostat.ec.europa.eu/portal/page/portal/euroindicators/labour_market/database (letzter Zugriff: 09.06.2010).

44 Vgl. den im Juli veröffentlichten McCarthy Report: Colm McCarthy/Donal McNally/Pat McLoughlin/Maurice O'Connell/William Slattery/Mary Walsh: Report of the Special Group on Public Service Numbers and Expenditure Programmes. Volume I, Dublin 2009.

45 Vgl. IBEC: A step on the road to recovery, 2009, abrufbar unter: http://www.ibec.ie/Sectors/Lisbon/ LisbonTreaty2009.nsf/vPages/Why_Yes a-step-on-the-road-to-recovery?OpenDocument (letzter Zugriff: 24.11.2009)

46 Ireland for Europe: Six Reasons to Vote Yes, 28.09.2009, abrufbar unter: http://media.irelandforeurope.ie/ ? $\mathrm{p}=331$ (letzter Zugriff: 24.11.2009).

47 Enda Kenny: A Yes to Lisbon is a Yes to Jobs - Kenny, 14.09.2009, abrufbar unter: http://www.finegael.org/ news/a/1071/article/ (letzter Zugriff: 14.10.2009). 
Vorteile der Grundrechtecharta herausstellte, das Demokratie-Argument, das die entsprechenden institutionellen Neuerungen als wichtige Schritte zur Bewältigung des demokratischen Defizits bewertete, oder die Betonung des außenpolitischen Gewichts, das die Europäische Union mit dem Lissabon-Vertrag in der Welt gewinne.

\section{Fazit: ein Deutungskampf unter veränderten Vorzeichen}

Nationale Referendumswahlkämpfe sind unerbittliche Deutungskämpfe, in denen wesentliche Elemente der jeweiligen politischen Kultur sowie gesellschaftliches Wissen aktualisiert und auf den Abstimmungsgegenstand angewandt werden. Wahrheiten der einen Seite werden mittels Vereinfachung und Zuspitzung im öffentlichen Diskurs zu Halbwahrheiten und kommen auf der anderen Seite als Lügen an. Am konkreten Beispiel Irlands lässt sich beobachten, wie zwei Abstimmungen zum weitgehend unveränderten Gegenstand, zwischen denen gerade einmal sechzehn Monate liegen, mit einem vollkommen unterschiedlichen Ergebnis endeten. Mit Blick auf die beteiligten Sprecher lassen sich im Großen und Ganzen nur geringe Veränderungen zum ersten Referendum 2008 feststellen: Im ,Nein'-Lager war die Organisation „Libertas“ zwar bei Weitem nicht so aktiv und wirkungsvoll wie 2008, ansonsten trat allerdings im Großen und Ganzen dasselbe Spektrum von Ratifizierungsgegnern an wie im Jahr zuvor. Auf der , Ja'-Seite spielten die bekannten Akteure eine aktivere Rolle als im Vorjahr und es kamen wichtige zivilgesellschaftliche Akteure wie insbesondere „Ireland for Europe" hinzu.

Was die Themen der Debatte betrifft, konnte das ,Nein'-Lager auf wichtige Argumente des letzten Jahres nicht mehr - Informationsmangel - oder wegen der Garantien nicht mehr in gleichem Ausmaß - sozialethische Kritik, Militarisierung, Steuerharmonisierung - zurückgreifen. Auf der anderen Seite fanden die Ratifizierungsbefürworter mit der Wirtschaftskrise und dem darauf ausgerichteten ökonomischen Argument ein bedeutendes Thema, mit dem sie die Debatte dominieren und auf die sozioökonomische Verunsicherung der Bevölkerung zielen konnten. Dieses wahltaktische Kalkül scheint seine Wirkung bei der Abstimmung nicht verfehlt zu haben. Zumindest ergibt eine Eurobarometerumfrage zu den Gründen für das , Ja' im zweiten irischen Referendum, für die Meinung, dass eine Zustimmung zum Vertragswerk der irischen Wirtschaft auf dem Weg der Erholung helfen könnte, einen Spitzenwert. ${ }^{48}$ Der von vielen Vertragsgegnern angesichts der Bedeutung des ökonomischen Arguments kritisierte Sieg der Angst ließe sich mithin von den Ratifizierungsbefürwortern ebenso gut als Sieg der Hoffnung interpretieren. Und dank ihrer konstruktivistischen Grundannahmen kann die vorliegende Untersuchung die Frage, welche Seite Recht hat, schließlich in diplomatischer Weise beantworten: zweifellos beide.

48 Europäische Kommission: Lisbon Treaty Post-Referendum Survey Ireland, 2009. 